\title{
Efficient Prediction of Temperature-dependent Elastic and Mechanical Properties of 2D Materials
}

Chinedu Ekuma ( $\nabla$ che218@lehigh.edu )

Lehigh University

Z Liu

Harbin Institute of Technology

Srihari Kastuar

Lehigh University

\section{Research Article}

Keywords: temperature-dependent elastic , 2D materials, materials design and discovery

Posted Date: September 16th, 2021

DOl: https://doi.org/10.21203/rs.3.rs-902313/v1

License: (c) (i) This work is licensed under a Creative Commons Attribution 4.0 International License.

Read Full License 


\title{
Efficient prediction of temperature-dependent elastic and mechanical properties of 2D materials
}

\author{
S. M. Kastuar, ${ }^{1}$ C. E. Ekuma, ${ }^{1, *}$ and Z. -L. Liu ${ }^{2,3}$ \\ ${ }^{1}$ Department of Physics, Lehigh University, Bethlehem, PA 18015, USA \\ ${ }^{2}$ School of Materials Science and Engineering, Harbin Institute of Technology, Harbin, China \\ ${ }^{3}$ College of Physics and Electric Information, Luoyang Normal University, Luoyang 471934, China
}

(Dated: August 24, 2021)

\begin{abstract}
An efficient automated toolkit for predicting the mechanical properties of materials can accelerate new materials design and discovery; this process often involves screening large configurational space in high-throughput calculations. Herein, we present the ElasTool toolkit for these applications. In particular, we use the ElasTool to study diversity of 2D materials and heterostructures including their temperature-dependent mechanical properties, and developed a machine learning algorithm for exploring predicted properties.
\end{abstract}

\section{INTRODUCTION}

The mechanical and elastic properties of materials are among the most fundamental properties that must be accurately determined and are essential in many disciplines not limited to condensed matter physics, materials science, and geophysics ${ }^{12}$ In new materials design and integration into devices, mechanical properties, and elastic constants are frequently used to determine the stability of the structures. ${ }^{3 / 4}$ The fundamental challenge in creating such new materials includes (i) the ability to enumerate the compositional space that is often too large for an Edisonian approach, and (ii) rapidly characterize the thermodynamic, electronic, and optical properties. To facilitate this process, there is a growing need to develop an automated computational toolkit for predicting essential properties such as the elastic and mechanical properties of bulk as well as 2D materials from firstprinciples elastic tensor calculations $\frac{[5] 6}{}$ Moreover, the accurate knowledge of the full elastic tensor enables the determination of many other important elastic, mechanical, and thermodynamic properties of materials that are essential for screening, design, and discovery of new materials 78

Recently, and specifically for 2D-based materials (Fig. 1), high-throughput elastic properties of several materials have been calculated and collected in some materials' databases such as the Materials Project, the JARVIS-DFT, and the Computational 2D Materials

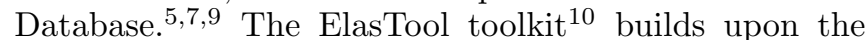
essential benefits of these already developed methods. However, unlike the existing toolkit for computing the elastic and mechanical properties of materials, the ElasTool toolkit can automate the process of computing zerotemperature as well as temperature-dependent elastic properties. It provides a complete elastic and mechanical toolkit for both automation and traditional prediction of zero-temperature, temperature-dependent, and the combined impact of dynamical pressure and temperature on crystal systems.

The zero-temperature elastic and mechanical properties of several 2D-based materials, including their het- erostructures and the less studied trichalcogenides, have been computed (see Table 1 in the Supplementary Material) ${ }^{11}$ The predicted elastic and mechanical properties are in excellent agreement with existing data in literature $\sqrt{518 \mid 12}\left[15\right.$. For example, our predicted $\mathrm{Y}^{2 D} \sim 135.86$ $\mathrm{N} / \mathrm{m}$ is in good agreement with recent experiments, which span from 129 to $185 \mathrm{~N} / \mathrm{m}^{14 \mid 16}[18$ and previous first-principles calculations that reported values 122 $146 \mathrm{~N} / \mathrm{m} \cdot \frac{19}{19}$ Our calculations show that most of the 1T-TMDs, e.g., 1T-MoSe ${ }_{2}$ exhibit an auxetic behavior, manifested by the negative Poisson ratio. This behavior has been observed in previous studies. ${ }^{20}$ It was attributed to the metastability of the 1T-TMDs mediated by the strong coupling between the chalcogen dominated $p$-states and the intermetallic $t_{2 g}$-bonding states within the basic triangular pyramid building block structure. Indeed, as shown in Table 2 (see Supplementary Material ${ }^{11}$ ), the application of temperature of $\sim 300 \mathrm{~K}$ stabilizes the auxetic states. Temperature-induced structural distortions in the $1 \mathrm{~T}$ structure (see Right Panel of Figure2 leading to a pressure of $\sim 2.41 \mathrm{~N} / \mathrm{m}$ (note the optimized vacuum size is $21.50 \AA$ ), which stabilized the structure. We also computed the elastic and mechanical properties of the less studied family of the transition metal trichalcogenides $\left(\mathrm{ABX}_{3}\right)$ with space $\mathrm{C} 3 \mathrm{i}$ (No. 147), where $\mathrm{A}=\mathrm{V}, \mathrm{Cr}, \mathrm{Mn}, \mathrm{Fe}, \mathrm{Co}, \mathrm{Ni}$, and $\mathrm{Cu} ; \mathrm{B}=\mathrm{Si}$ and $\mathrm{Ge}$; and $\mathrm{X}=\mathrm{S}$, Se, and Te. They form sandwich layers of X-(A,B)-X stacks. Each of the B atoms possesses three neighboring $\mathrm{X}$ atoms forming a tetrahedron, and two of the B-centered tetrahedrons forming a dumbbelllike $\mathrm{B}$ dimer in $\left[\mathrm{AX}_{6}\right]^{4-}$ bipyramid leading to a monolayer unit cell composed of two $\mathrm{A}^{2+}$ and one $\left[\mathrm{AX}_{6}\right]^{4-}$ ions. The structure is such that in-plane interactions are dominated by strong covalent bonds while interlayers are mediated by weak van der Waals interactions. The trichalcogenides, especially those with the $3 d$ end members, seem more stable with a spin-polarized solution. We note that even though $\mathrm{CrSiS}_{3}$ shows instability, spinpolarized solution stabilizes the mechanical properties with $\mathrm{K} \sim 196.43 \mathrm{~N} / \mathrm{m}, \mathrm{G} \sim 106.32 \mathrm{~N} / \mathrm{m}, \mathrm{Y}^{2 D} \sim 275.99$ $\mathrm{N} / \mathrm{m}$.

Hardness is a fundamental property of a material that 


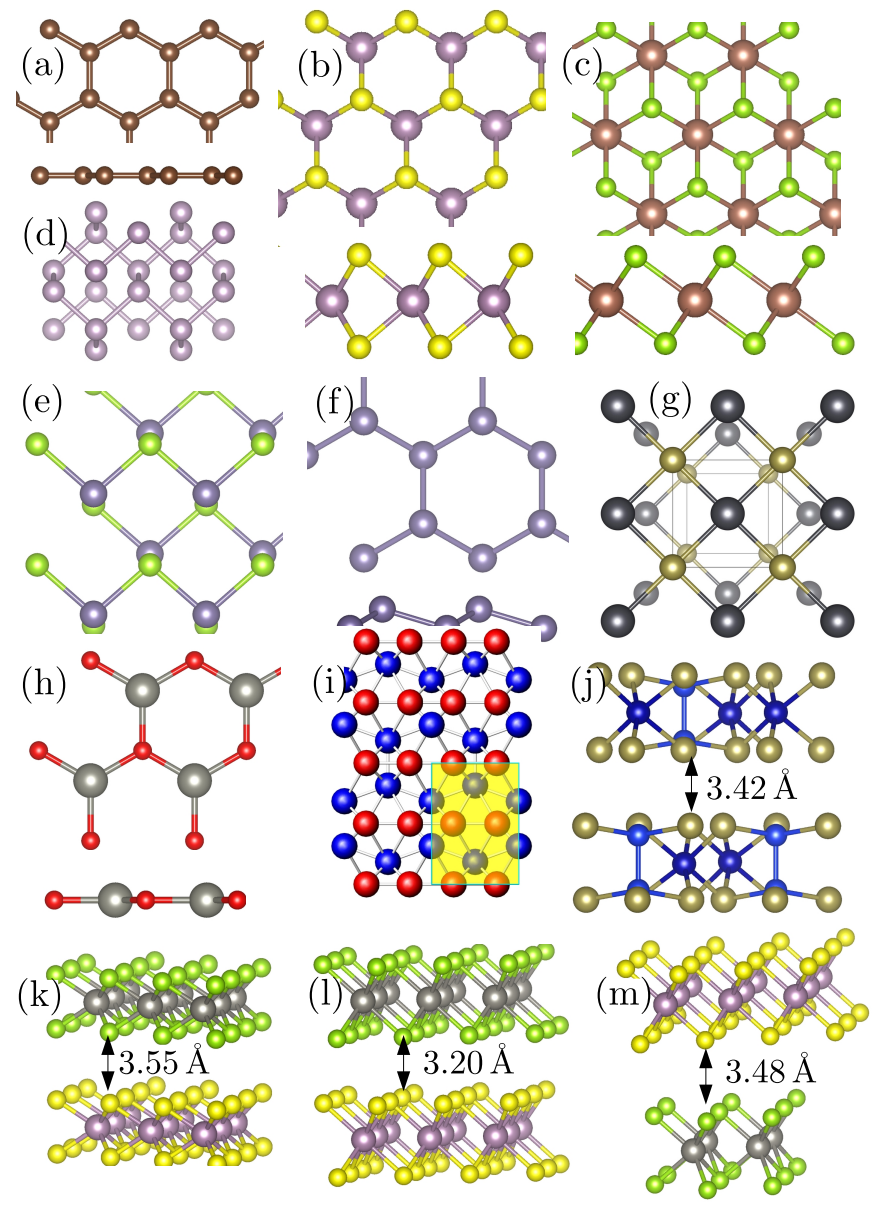

FIG. 1. Crystal structure of diversity of 2D-based materials and their heterostructures. (a) Top and side view of the hexagonal structure of graphene. (b) Top and side view of the trigonal prismatic crystal structure of $2 \mathrm{H}$ transition metal dichalcogenides (TMDs) such as $\mathrm{MoS}_{2}$. (c) Top and side view of the octahedral 1T-TMDs structures such as $\mathrm{WS}_{2}$ and posttransition metal chalcogenides such as $\mathrm{SnS}_{2}$. (d) Simple orthorhombic (Pmna, space group 53) structure of phosphorene. (e) Orthorhombic (Pmn2 1 , space group 7) structure of group IV monochalcogenides, e.g., GeSe. (f) Top and side view of the silicene-like structures. (g) Tetragonal crystal structure of group IV-VI monochalcogenides such as PbTe, (h) crystal structure of the IV-VI materials, e.g., ZnS, (i) 8-Pmmn orthorhombic crystal structure (highlighted area depicts the unit cell) of borophene. Blue (inner position atoms) and red (atoms along the ridges) depict the nonequivalent boron atoms. (j) Trigonal crystal structure of transition metal trichalcogenides $\mathrm{ABX}_{3}$, where $\mathrm{A}=\mathrm{V}, \mathrm{Cr}, \mathrm{Mn}, \mathrm{Fe}, \mathrm{Co}, \mathrm{Ni}$, and $\mathrm{Cu} ; \mathrm{B}=\mathrm{Si}$ and $\mathrm{Ge}$; and $\mathrm{X}=\mathrm{S}$, Se, and Te. $(\mathrm{k}-\mathrm{m})$ are the crystal structures of $2 \mathrm{H}, 1 \mathrm{~T}$, and $2 \mathrm{H}-1 \mathrm{~T}$ heterostructures designed with $\mathrm{MoS}_{2}$ and $\mathrm{WS}_{2}$, respectively.

plays an essential role in the full description of the mechanical properties of materials. To gain a fundamental understanding of the macroscopic mechanical properties of materials under deformation, the ideal strength, which is the minimum stress needed to plastically deform a material vis-à-vis the upper bound to the critical stress for dislocation and crack nucleation in a material can in principle be calculated. However, for high-throughput calculations in materials screening and new materials design, such calculations can be highly challenging due to the large configurational space. We can estimate the ideal strength based on the modification of empirical relations between elastic moduli and the Vickers hardness $\mathrm{H}_{v}$ proposed for bulk crystals. ${ }^{2122}$ Specific for 2D materials, we have the following empirical relations: $H_{v}^{1 a}=0.151 G, H_{v}^{1 b}=0.0608 Y^{2 D}, H_{v}^{1 c}=0.0963 \mathrm{~K}$, $H_{v}^{2}=0.1769 G-2.899, H_{v}^{3}=(1-2 \nu) K /(6+6 \nu)$, $H_{v}^{4}=2\left(G^{3} / K^{2}\right)^{0.585}-3$. To determine the best empirical model, we compute the hardness of the various $2 \mathrm{D}$ materials and heterostructures. Our test shows that all the models perform well for the superhard and moderately hard materials such as graphene, hexagonal BN, and borophene. For example, the $H_{v}$ for graphene using the models is $\sim 21 \pm 2 \mathrm{~N} / \mathrm{m}$ and $H_{v} \sim 16 \pm 1$ for hexagonal $\mathrm{BN}$, which is in good agreement with experiments if we use a thickness of approximately $3.35 \AA .23124$ However, aside from the superhard and moderately hard materials, our calculations show that models $H_{v}^{1 a-1 c}$ are robust enough to predict the hardness of the diversity of 2D materials and heterostructures. Specifically for the trichalcogenides, models $H_{v}^{1 a-1 b}$ are the most appropriate. In all cases, models $H_{v}^{2-4}$ are highly unreliable with very large uncertainties and should be avoided for 2Dbased materials.

Machine learning (ML) is a burgeoning approach in diverse fields to unravel hidden structure-property relations. To gain a deeper understanding of the relation between the computed elastic and mechanical properties, we develop ML-trained models for the relevant features (see all the details and accompanying figures in the Supplementary Material). After an initial training of the ML algorithm, we employed hyperparameter optimization, which systematically finds the optimal set of values for the parameters that control the learning process and the performance of a ML algorithm. Additionally, we implemented a 5 -fold cross-validation that is essential to reduce the potential of overfitting while fully utilizing the breadth of the dataset obtained with first-principles calculations. Our ML-trained model led to an average $\mathrm{R}^{2}$ value of 0.97 .

To summarize, we present an efficient computational toolkit based on density functional theory and ab-initio molecular dynamics to automate the calculation of the elastic and mechanical properties of several 2D materials and their heterostructures at both zero- and finitetemperature. A machine learning algorithm is developed for exploring the relation between the predicted properties. 

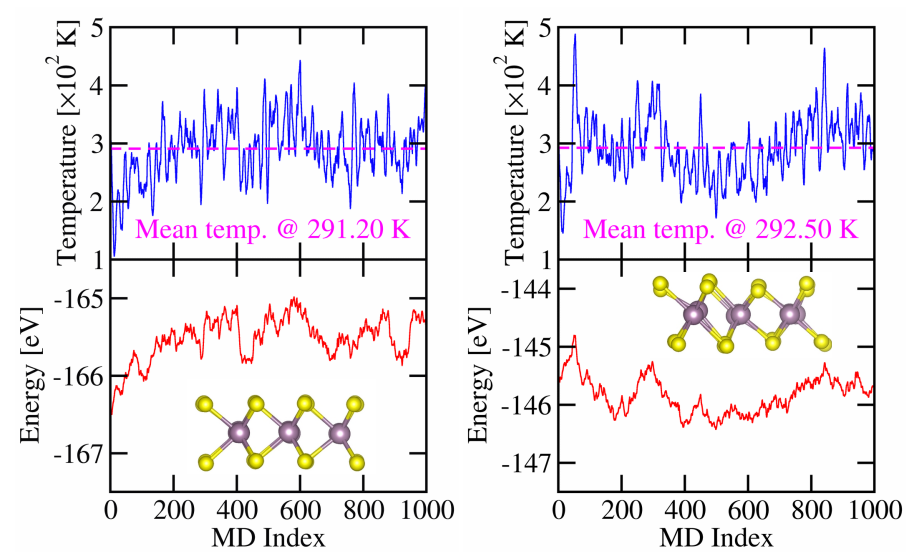

FIG. 2. A representative profile of temperature and the total energy at various molecular dynamics steps. Left panel is for $2 \mathrm{H}-\mathrm{MoS}_{2}$ and right panel is for $1 \mathrm{~T}-\mathrm{MoS}_{2}$. The inset in both plots is the crystal structure at the simulated temperature of $300 \mathrm{~K}$. The applied temperature induced $0.0(2.41 \mathrm{~N} / \mathrm{m})$ pressure on the $2 \mathrm{H}(1 \mathrm{~T}) \mathrm{MoS}_{2}$ crystal structures.

\section{METHOD}

Theoretical background. The stress-strain and the strain-energy method are the two essential approaches to compute the elastic properties of materials. ${ }^{[25|26|}$ While the stress-strain approach is computationally more expensive, it is, however, simpler to implement and does not require the complicated pressure corrections needed for the strain-energy method when computing high-pressure elastic constants of materials. 27 Under a small and homogeneous deformation $\mathbf{D}=\mathcal{I}+\xi$, where $\mathcal{I}$ is a $3 \times 3$ unit matrix. Within the elastic limit, the generalized stress-strain Hooke's law is $\sigma_{i j}=C_{i j k l} \xi_{k l}$, where $C_{i j k l}$ is a fourth-rank elastic tensor and $\sigma_{i j}\left(\xi_{k l}\right)$ is a second-rank stress (strain) tensor. In Voigt notation, the stress-strain relation is

$$
\sigma_{i}=\sum_{j=1}^{6} C_{i j} \xi_{j}
$$

For 2D materials, only the in-plane elastic matrix is essential $13 \mid 28$

$$
\left[\begin{array}{l}
\sigma_{1} \\
\sigma_{2} \\
\sigma_{6}
\end{array}\right]=\left[\begin{array}{ccc}
C_{11} & C_{12} & 0 \\
C_{12} & C_{22} & 0 \\
0 & 0 & C_{66}
\end{array}\right]\left[\begin{array}{l}
\xi_{1} \\
\xi_{2} \\
\xi_{6}
\end{array}\right]
$$

where $C_{66}=\left(C_{i i}-C_{i j}\right) / 2, i=1,2$. After deformation, the crystal lattice vector can be obtained as $\mathbf{A}^{\prime}=\mathbf{A} . \mathbf{D}$, where $\mathbf{A}$ is the undeformed crystal lattice vector. The elastic constants can readily be obtained by a polynomial fit of Eq.2. Having obtained the elastic constants, we can compute other related properties. The in-plane arrangement of 2D-material designates the atomic arrangement in an $x-y$ plane of chirality angle $\theta$ that span between
0 and $30^{\circ}$, where $\theta=0^{\circ}$ and $30^{\circ}$ are for the zigzag and armchair chirality, respectively. For any arbitrary angle, the in-plane (2D) Young's modulus $\mathrm{Y}^{2 D}$ and the Poisson's ratio $\nu$ are 29

$$
\begin{gathered}
Y^{2 D}(\theta)=\frac{C_{11} C_{22}-C_{12}^{2}}{C_{11} s^{4}+C_{22} c^{4}+c^{2} s^{2} \gamma} \\
\nu(\theta)=\frac{C_{11} C_{22}-\frac{C_{11} C_{22}-C_{12}^{2}}{C_{66}} c^{2} s^{2}-C_{12}\left(c^{4}+s^{4}\right)}{C_{11} s^{4}+C_{22} c^{4}+c^{2} s^{2} \gamma}
\end{gathered}
$$

where $\gamma=\left[\left(C_{11} C_{22}-C_{12^{2}}\right) / C_{66}-2 C_{12}\right] c^{2} s^{2}, c=\cos \theta$, and $s=\sin \theta$. Assuming $\theta=0$, then

$$
Y^{2 D}=\frac{C_{i i}^{2}}{C_{i j}^{2}} ; \quad \nu=\frac{C_{i j}}{C_{i i}} \quad ; i, j=1,2
$$

Having obtained the Young's modulus and the Poisson ratio, we can compute the in-plane stiffness (layer modulus) $K$, i.e., the $2 \mathrm{D}$ equivalent of the bulk modulus and the shear modulus $\mathrm{G}$ as 30

$$
K=\frac{Y^{2 D}}{2(1-\nu)} ; \quad G=\frac{Y^{2 D}}{2(1+\nu)} .
$$

The bulk modulus measures the resistance of a bulk material to compression while the layer modulus represents the resistance of a $2 \mathrm{D}$ material to stretching. Stiffness is a measure of the resistance of a material to elastic deformation. We can use it to measure the sound velocity in crystals $[62,63]$ as

$$
V_{l}=\sqrt{\frac{K+G}{\rho_{2 D}}} ; \quad V_{t}=\sqrt{\frac{G}{\rho_{2 D}}}
$$

where $V_{l}$ and $V_{t}$ are the longitudinal and shear sound velocity, respectively, and $\rho_{2 D}$ is the $2 \mathrm{D}$ mass density. We note that $V_{t}$ has also been defined as $V_{t}=\sqrt{C_{12} / \rho_{2 D}}$ Having obtained the sound velocities, it is straightforward to compute the Debye temperature $\Theta_{D}$ from the average sound velocities $V_{a}$ as 32

$$
\Theta_{D}=\frac{\hbar V_{a}}{\kappa_{B}}\left(\frac{4 \pi N}{S}\right)^{1 / 2}
$$

where $\hbar$ is the reduced Planck constant, $N$ is the number of atoms in the unit cell, $\kappa_{B}$ is the Boltzmann constant, $S$ is the area of the unit cell, and $V_{a}$ for a typical $2 \mathrm{D}$ system is

$$
V_{a}=\left[\frac{1}{2}\left(\frac{1}{V_{l}^{2}}+\frac{1}{V_{t}^{2}}\right)\right]^{-1 / 2}
$$

Code structure. The ElasTool toolkit is based on Python and can be downloaded from GitHub!10 It exploits the versatility of several Python-based libraries 
such as NumPy for numerical calculations, ASE for structure manipulations, Pandas for efficient statistics of stress tensor calculations, and Spglib for automatic determination of the crystal structure symmetry. These libraries are easily installed via pip or conda. Currently, the ElasTool is interfaced with the VASP electronic structure code; interfacing with other electronic structure codes is straightforward. The main input file named "elastool.in" is needed to run the code. The crystal information is provided in the standard crystal information format or the POSCAR format, which is the standard form for VASP code. Other key input parameters are self explanatory and require no technical knowledge. Since the ElasTool code is currently interfaced with VASP, various key files namely INCARs for structural optimization and stress tensor calculations, KPOINTS-static for zero-temperature calculations, KPOINTS-dynamic for temperature-dependent calculations using $a b$ initio molecular dynamics are provided. While the code usage is easy and straightforward, we have also provided several example calculations in the example folder.

Computational details. The ElasTool toolkit obtains the second-order elastic constants of any material using highly optimized, high-efficient strain-matrix sets. It currently uses the VASP code $\sqrt{33}$ as the stress-strain calculator. Extension to other electronic structure codes is straightforward. All the calculations were done within the first-principles density functional theory ${ }^{34}$ using the Perdew-Burke-Ernzerhof (PBE) $)^{\sqrt{35}}$ exchange-correlation functional. The k-point sampling was done on a $15 \times 15 \times$ $1 \Gamma$-center grid. The total energy (charge) is converged to within $10^{-7}\left(10^{-3}\right) \mathrm{eV}$, with the residual stresses and forces less than $0.01 \mathrm{~N} / \mathrm{m}$ and $10^{-3} \mathrm{eV} / \AA$ using a Fermi distribution function with a smearing parameter of $0.10 \mathrm{eV}$ to integrate the states at the Fermi level. In all the cases, a kinetic energy cutoff of $550 \mathrm{eV}$ was used. All calculations included van der Waals interaction corrections to avoid spurious interactions between the periodically repeated images of the slab. We used a vacuum of at least $15 \AA$ along the out-of-plane direction to further eliminate the artifacts of the periodic boundary condition. The temperature-dependent elastic and mechanical properties were obtained using the thermal stress-strain data from $a b$ initio molecular dynamics (AIMD) ${ }^{36}$ as implemented in VASP 33 . These calculations employed $5 \times 5 \times 1 \Gamma$-center grid to sample the Brillouin zone. AIMD provides a reliable description of the time-evolution of systems and often reveals non-intuitive temperature-dependent system configurations. The structures were initially optimized with the PBE exchange-correlation functional and then equilibrated under an isothermal isobaric (NPT) ensemble using the Langevin thermostat to maintain the temperature. The structures were further simulated under a canonical (NVT) ensemble using the Nose-Hoover thermostat to maintain the temperature. All calculations used cutoff energy of $550 \mathrm{eV}$, a time step of $2 \mathrm{fs}$, and 1000 molecular dynamics (MD) steps with the last 500 MD steps used to average the thermal stresses. A representative $\mathrm{MD}$ profile of the temperature of the total energy for $2 \mathrm{H}$ - and $1 \mathrm{~T}-\mathrm{MoS}_{2}$ are shown in Figure 2 .

\section{DATA AVAILABILITY}

All data produced and analyzed in the this study are included in this published article. Additionally, we have computed the elastic and mechanical properties of over ten thousand 2D materials and their heterostructures. We will continue to update the database and the corresponding machine learning algorithm in the GitHub repository https://github.com/gmp007/ 2D_Elastic-Properties. Additional correspondence and request can be made by email to the authors.

\section{REFERENCES}

* che218@lehigh.edu

1 N. Ashcroft and N. Mermin, Solid State Physics, HRW international editions (Holt, Rinehart and Winston, 1976), ISBN 9780030839931.

2 S. Crampin, Geophysical Journal International 76(1), 135145, ISSN 0956-540X (1984).

3 M. Born, Mathematical Proceedings of the Cambridge Philosophical Society 36(2), 160-172 (1940).

${ }^{4}$ M. Born and K. Huang, Dynamical Theory of Crystal Lattices, International series of monographs on physics (Clarendon Press, 1988), ISBN 9780198503699.

5 S. Haastrup, M. Strange, M. Pandey, T. Deilmann, P. S. Schmidt, N. F. Hinsche, M. N. Gjerding, D. Torelli, P. M. Larsen, A. C. Riis-Jensen, J. Gath, K. W. Jacobsen, J. J. Mortensen, T. Olsen, and K. S. Thygesen, 2D Materials 5(4), 042002 (2018).
6 J. Zhou, L. Shen, M. D. Costa, K. A. Persson, S. P. Ong, P. Huck, Y. Lu, X. Ma, Y. Chen, H. Tang, and Y. P. Feng, Scientific Data 6(1), 86, ISSN 2052-4463 (2019).

7 M. de Jong, W. Chen, T. Angsten, A. Jain, R. Notestine, A. Gamst, M. Sluiter, C. Krishna Ande, S. van der Zwaag, J. J. Plata, C. Toher, S. Curtarolo, G. Ceder, K. A. Persson, and M. Asta, Scientific Data 2(1), 150009, ISSN 2052-4463 (2015).

8 S. Chibani and F.-X. Coudert, Chem. Sci. 10, 8589-8599 (2019).

9 K. Choudhary, G. Cheon, E. Reed, and F. Tavazza, Phys. Rev. B 98, 014107 (2018).

10 Z.-L. Liu and C. E. Ekuma, Elastool: An automated Python-based toolkit for calculating elastic constants, GitHub: https://github.com/zhongliliu/elastool (2020); Z.-L. Liu and C. E. Ekuma, Elastool: An automated toolkit for elastic constants calculation (2020). 
11 See Supplemental Material at [URL will be inserted by publisher] of the elastic and mechanic properties for the various $2 \mathrm{D}$ materials and their heterostructures. Also presented are the details of the machine learning algorithm

${ }^{12}$ D. Akinwande, C. J. Brennan, J. S. Bunch, P. Egberts, J. R. Felts, H. Gao, R. Huang, J.-S. Kim, T. Li, Y. Li, K. M. Liechti, N. Lu, H. S. Park, E. J. Reed, P. Wang, B. I. Yakobson, T. Zhang, Y.-W. Zhang, Y. Zhou, and Y. Zhu, Extreme Mechanics Letters 13, 42 - 77, ISSN 2352-4316 (2017).

13 X. Wei, B. Fragneaud, C. A. Marianetti, and J. W. Kysar, Phys. Rev. B 80, 205407 (2009).

14 C. Andres, P. Menno, S. G. A., van der Zant Herre S. J., A. Nicolás, and R. Gabino, Advanced Materials 24(6), 772-775 (2012).

15 G. Casillas, U. Santiago, H. Barrón, D. Alducin, A. Ponce, and M. José-Yacamán, The Journal of Physical Chemistry C 119(1), 710-715, pMID: 25598860 (2015).

16 K. Liu, Q. Yan, M. Chen, W. Fan, Y. Sun, J. Suh, D. Fu, S. Lee, J. Zhou, S. Tongay, J. Ji, J. B. Neaton, and J. Wu, Nano Letters 14(9), 5097-5103, pMID: 25120033 (2014).

17 A. Castellanos-Gomez, M. Poot, G. A. Steele, H. S. van der Zant, N. Agraït, and G. Rubio-Bollinger, Nanoscale Research Letters 7(1), 233, ISSN 1556-276X (2012).

18 R. C. Cooper, C. Lee, C. A. Marianetti, X. Wei, J. Hone, and J. W. Kysar, Phys. Rev. B 87, 035423 (2013).

19 T. Li, Phys. Rev. B 85, 235407 (2012).

${ }^{20}$ L. Yu, Q. Yan, and A. Ruzsinszky, Nature Communications 8(1), 15224, ISSN 2041-1723 (2017).

21 X. Jiang, J. Zhao, and X. Jiang, Computational Materials Science 50(7), 2287-2290, ISSN 0927-0256 (2011).

22 D. M. Teter, MRS Bulletin 23(1), 22-27 (1998).

23 J. P. Lu, Phys. Rev. Lett. 79, 1297-1300 (1997).

24 D. Golla, K. Chattrakun, K. Watanabe, T. Taniguchi, B. J. LeRoy, and A. Sandhu, Applied Physics Letters 102(16), 161906 (2013).

25 R. Golesorkhtabar, P. Pavone, J. Spitaler, P. Puschnig, and C. Draxl, Computer Physics Communications 184(8), 1861-1873, ISSN 0010-4655 (2013).

${ }^{26}$ U. Jindal, Strength of Materials: Always learning (Pearson Education India, 2012), ISBN 9789332501232.

27 G. V. Sin $\$$ rquot\$ko and N. A. Smirnov, Journal of Physics: Condensed Matter 14(29), 6989-7005 (2002).

28 J. Zhou and R. Huang, Journal of the Mechanics and Physics of Solids 56(4), 1609 - 1623, ISSN 0022-5096 (2008).

29 E. Cadelano, P. L. Palla, S. Giordano, and L. Colombo, Phys. Rev. Lett. 102, 235502 (2009).
30 A. Politano and G. Chiarello, Nano Research 8(6), 18471856, ISSN 1998-0000 (2015).

31 L. Kinsler, A. Frey, A. Coppens, and J. Sanders, Fundamentals of Acoustics (Wiley, 1982), ISBN 9780471029335.

32 H. Shao, X. Tan, J. Jiang, and H. Jiang, EPL (Europhysics Letters) 113(2), 26001 (2016).

33 G. Kresse and J. Furthmüller, Comput. Mater. Sci. 6(1), 15-50 (1996).

34 P. Hohenberg and W. Kohn, Phys. Rev. 136, B864-B871 (1964); W. Kohn and L. J. Sham, Phys. Rev. 140, A1133A1138 (1965).

35 J. P. Perdew, K. Burke, and M. Ernzerhof, Phys. Rev. Lett. 77, 3865-3868 (1996).

36 R. Car and M. Parrinello, Phys. Rev. Lett. 55, 2471-2474 (1985).

\section{ACKNOWLEDGEMENT}

S.M.K. acknowledges the Lee graduate fellowhsip. C.E.E acknowledges start-up grant and summer research grants from Lehigh University (LU). Computational resources are provided by the LU HPC Center. Z.L.L acknowledges the support from the Key Research Scheme of Henan Universities under grant No. 18A140024

\section{CODE AVAILABILITY}

The ElasTool is available free of charge on GitHub ${ }^{[0]}$ Developmental version can also be requested directly by emailing the authors.

\section{COMPETING INTEREST}

The authors declare no competing interest.

\section{AUTHOR CONTRIBUTIONS}

C.E.E proposed and designed the study, Z.L.L. and C.E.E. wrote the ElasTool code, S.K. and C.E.E. wrote the machine learning algorithm, C.E.E. performed the simulations and data analysis. All the authors discussed and analyzed the results and participated in writing the present manuscript. 


\section{Supplementary Files}

This is a list of supplementary files associated with this preprint. Click to download.

- 2DElasticSupplemental.pdf 